\section{J.Kraft Microscopy Services, Inc.}

J.Kraft Microscopy Services, Inc. was founded in 2012. Since then our mission has been to help our customers recognize their research and laboratory development goals. We are dedicated to ensuring that we are giving our customers the knowledge and attention needed for them to make informed decisions about the equipment they have now and are purchasing in the future.

Combining years of experience across multiple brands, a unique catalog of products, and a consultative approached to developing solutions, J.Kraft Microscopy has the tools to help. Working with our current customers they have been able to reduce costs, increase capabilities, and focus more on obtaining the results they need.

Through our strong working relationships with key industry leaders and our distribution partnership with Point Electronic GmbH; J.Kraft Microscopy can offer you the highest level of service, fast turnaround times, affordable equipment, and cutting edge technology.

- SEM Upgrade-Replace your systems current electronics with new cutting-edge hardware and software, including control of all electron-optics on the column, electron gun power supply, all electron detectors, sample stage and chamberscope. • Powerful and versatile, the DISS5 image acquisition system from Point Electronic, puts all your scanning and acquisition controls in one easy to use program. Scan at faster speeds, achieve greater control and obtain multiple imaging signals simultaneously. Easily share your data with Windows 10 and networking capability.

- SEM Topography-Complete hardware and software package that utilizes conventional BSE signals to generate 3D topography in real-time. - Generate and analyze 3D models allowing you to understand you samples differently, connect a 3D printer to your system and print your samples for greater audience impact.

- EBIC acquisition-Sophisticated and easy to use EBIC amplifier, offering complete software control of all amplification and acquisition settings. - Develop custom workflows with configurable scan profiles, for increased efficiency and performance.

- EBAC/RCI acquisition-Completely software controlled for efficient and convenient use, image scanning and amplifier control are integrated into one application - Create customer workflows to quickly and accurately pinpoint contamination, defects, and resistive interconnectors.

- SERVICE-With a robust network of experienced and highly trained professionals, your system can be serviced anywhere in the U.S., or around the globe. - Responsive and costeffective service options, we offer preventative maintenance, upgrades, repair, and complete refurbishing options.

- Microscope Moving-Complete Turnkey electron microscope moving service. Relocating an electron microscope requires following specific procedures to avoid damage and ensure a safe, seamless transition to its new location. Let our highly trained experts help you.

- Electron Microscopes-Professionally refurbished and tested systems are available in stock, ready to go into service in a variety of applications. - Looking for Great performance on a budget, we also offer completely upgraded SEM systems. With new electronics and software from Point Electronic, these systems offer state of the art control, performance and reliability for years to come at a price point that is more affordable than a brand-new system. - Dedicated to finding the right electron microscope, J.Kraft Microscopy will work with you to determine what systems will fit your needs. If we don't have the right system in stock, we will use our extensive market knowledge to help you find it.

Originally started as a service company, J.Kraft Microscopy Services, Inc, has grown under the guidance of its founder Justin Kraft, to provide more. We continue to build new relationships and introduce new products to our catalog that focus on providing high quality and cost-effective options for your microscopy needs.
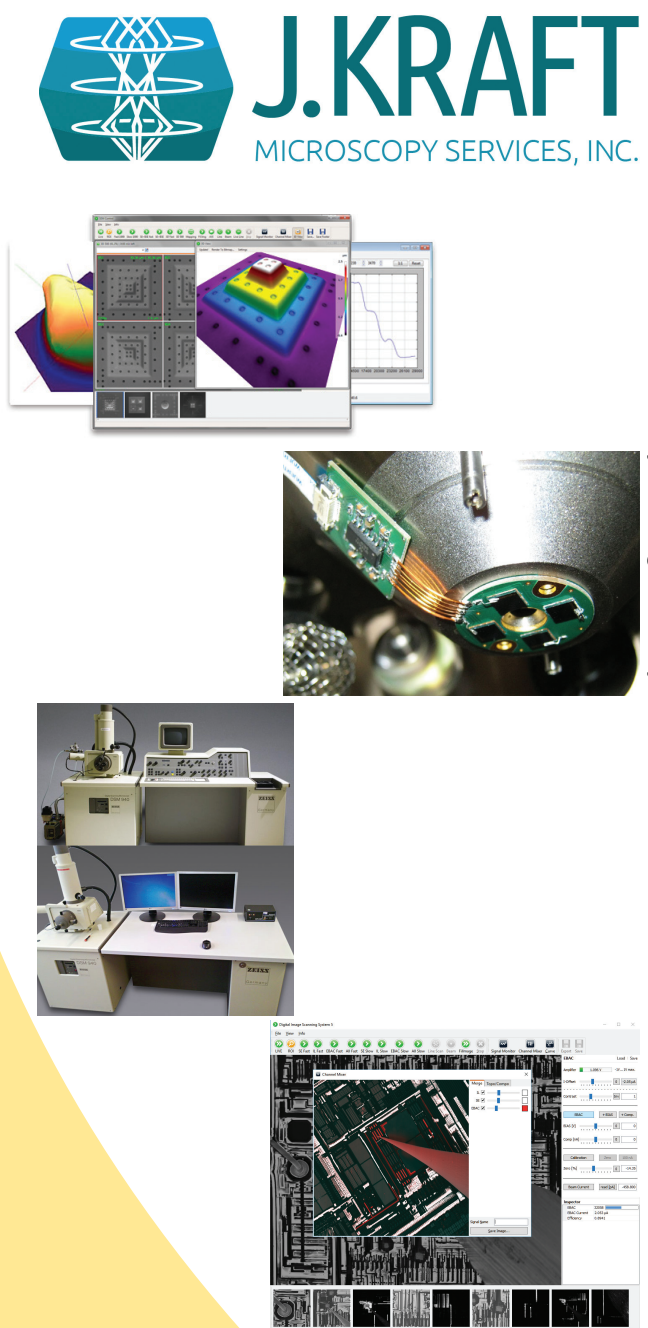

\section{How to find us}

\author{
J.KRAFT MICROSCOPY SERVICES, INC. \\ 243 Main St PO BOX 386 \\ Springville, NY, 14141 \\ Tel: 716-592-4402 \\ Fax: 716-592-4407 \\ Email: Info@Jkraftmicro.com \\ www.jkraftmicro.com
}

FRANCE +330975181013 